Vol. 6, Num. 1, 2021

\title{
INVESTIGATION OF GREENHOUSE MONITORING AND CONTROL SYSTEM
}

\author{
Tetiana Sahaidak, Nataliia Huzynets \\ Lviv Polytechnic National University, 12, Bandera Str, Lviv, 79013, Ukraine. \\ Authors'e-mail:tetiana.sahaidak.ki.2017@lpnu.ua,natdemchuk@ukr.net
}

https://doi.org/10.23939/acps2021.01.

Submitted on 09.05.2021

(C) Sahaidak T., Huzynets N., 2021

\begin{abstract}
Unsuitable climatic conditions, various natural disasters and instability and unpredictability of the weather significantly complicate cultivation, and sometimes make it even impossible. To ensure the best conditions for cultivation and the highest yields, farmers began to use greenhouses. However, in our hectic lives, people are constantly busy with something and there is no enough time. Long trips, business trips, vacations are also possible. It is becoming increasingly difficult to provide the necessary conditions for plants to grow on their own. That is why the Internet of Things has been so successfully integrated with agriculture that it has led to the emergence of automated or intelligent greenhouses.

The article attempts to analyze the types of greenhouse monitoring and control system, their technical characteristics, principles of operation and basic requirements for these systems. According to the results of the study, the best smart greenhouses have been selected. The main functions of automated greenhouses have been described. Selection criteria have been determined and a comparative analysis of the most popular products available on the market.
\end{abstract}

Index Terms: IoT, smart greenhouse, sensors, smart farming, greenhouse farming, agriculture

\section{INTRODUCTION}

From ancient times the people began to grow plants. At first it was due to the need to get more food, but later the cultivation of ornamental plants, such as flowers, began to spread. For normal growth, plants need certain conditions: sufficient lighting, soil moisture, a certain ambient temperature environment, the presence of air components in a certain percentage quantity (oxygen, carbon dioxide). All this leads to necessity care for them, especially if they are exotic plants that need specific conditions not inherent in the climatic zone in which they want grow.

In our hectic live, people are constantly busy with something and time is extremely lacking. Long trips, business trips, vacations are also possible. All this time it is necessary to provide the correct watering of plants, to watch, so that they receive the required amount of light, especially in winter when daylight is the shortest. It is extremely important to maintain the required temperature for a particular plant species, because it can die at too low or too high temperature. It should be noted that to maintain a constant temperature it is necessary to build walls around plants, and this creates the next problem - the lack of air circulation and carbon dioxide deficiency or surplus (depending on the situation). So it is also necessary to provide the plant with air from the environment that contains the required number of components in percent relation to each other. To solve all the above problems, there are smart greenhouses.

Internet of Things - the concept of a data network between physical objects, equipped with built-in tools and technologies for interaction with each other or with the external environment. This industry was born in the 1980s, but only now we have the first significant results. People try to give the "mind" of literally everything around: household appliances, industrial, transport, even whole houses and structures.

IoT plays an extremely important role in agriculture. It can help farmers reduce waste and improve productivity. For example, studies show that as much as $60 \%$ of irrigation water is wasted. A smart irrigation system can collect data on soil conditions and plant needs to selectively irrigate different plots of land. 14 bee farms have announced a $40 \%$ reduction in water use. The data can also be combined with the weather forecast and delay irrigation if rain is expected. Smart farms can also benefit from other types of smart facilities. "Smart" garbage containers and silo towers can report the level of grain and other feed they contain, to simplify management and avoid risky inspections themselves. These devices can also send warnings when the temperature in the containers rises to a level that could damage or degrade their contents.

\section{THE RATIONALE OF THE NEED FOR GREENHOUSE MONITORING AND CONTROL SYSTEM}

Greenhouse - a structure for growing plants that require special climatic conditions. The first idea of growing plants in a climate-controlled environment originated in the days of the Roman Empire and has developed to this day. Today, greenhouses have become widespread due to population growth, climate change and 
urbanization. The growth of their demand over the last 5 years is especially noticeable. Their undoubted advantage is the ability to harvest several times throughout the year. Greenhouses differ in size, which also allows their use not only by entrepreneurs but also by summer residents or ordinary apartment dwellers. Not only cultivated plants but also ornamentals are grown here.

Smart greenhouse - a revolution in agriculture, which creates the necessary microclimate for plants, which is automatically regulated through the use of sensors, actuators and control systems. This design has completely changed the standard approaches to growing plants.

Using the Internet of Things in agriculture significantly saves time, money and labor [1]. The growth in demand for this design is due to the presence of four main advantages:

1. Data collection from sensors. Smart greenhouses are equipped with many sensors. For example, the sensor of air temperature, humidity, soil moisture, soil salinity, lighting and many others. All of them provide detailed and accurate information about the conditions in which plants are grown. This makes it easy to control and maintain the most favorable microclimate.

2. Remote data access. Imagine that a farmer needs to go somewhere for a while, but he can't leave his greenhouse at random. He still needs to control all the important parameters. Smart greenhouses collect all the data and send it to a specific mobile application, website or cloud platform where the farmer can authenticate and have all the necessary information in real time.

3. Automated equipment control. Process automation saves time, increases the ability to control in critical situations and reduces the need for human labor. Everything works independently. For example, if the soil moisture is too low, the motor starts, which irrigates the plants.

4. Data analysis. Sensors installed in the greenhouse provide all the necessary information about the environment. Analysis of this data with the help of machine learning technologies helps to make better decisions about the growing conditions of plants and get a better harvest.

\section{FORMULATION OF THE PROBLEM}

The modern market of smart greenhouses does not yet offer the buyer a great variety of systems, as this industry has just begun to develop. Systems with fully automated basic functions are currently only available to owners of greenhouse complexes. If you consider the systems for individual use - they all offer very little functionality at a very high price. This is also due to the fact that smart minigreenhouses have only recently appeared.

The task of the work is to determine the requirements for smart greenhouses, as well as to conduct a comparative analysis of the characteristics of existing systems for their compliance with these requirements to determine those that best meet the requirements.

\section{IV.FUNSCTIONS AND FUNCTIONAL PRINCIPLES OF THE SMART GREENHOUSES}

Equipped with modern sensor technology, smart greenhouses provide round-the-clock information on the microclimate and harvest. The collected data can be analyzed by the IoT platform and automatically adjusted in case of deviations from the set norm. It is also possible to perform certain operations, such as watering or ventilation, on demand at the touch of a button. Continuous monitoring of data contributes to the development of predictive models for assessing the risks of crop diseases and infection.

Maintenance of ideal microclimatic conditions.

Providers collect data in unprecedented detail and provide farmers with real-time information on key climatic factors. This data is analyzed and adjusted according to predefined HVAC and lighting settings, providing plants with the most comfortable conditions for growth and energy efficiency.

The main monitored indicators are humidity and air temperature and plant lighting. Temperature and humidity control is one of the most important functions of the greenhouse, because they have perhaps the greatest impact on plant growth and yield. This data is most often collected using a temperature and humidity sensor. Light is involved in the photosynthesis of plants, measured by a light sensor.

Fully automated greenhouses not only allow you to control climatic parameters, but also automatically maintain them. One can maintain the temperature within acceptable limits by air conditioning or heating. Air conditioning is also important for photosynthesis and respiration of plants, as well as prevents the accumulation of pathogens that like stagnant air. Lowering the air temperature can be done by simple ventilation. Actuators open a window when the temperature reaches the upper critical limit (only if the outside temperature is lower). The smart greenhouse also automatically turns on the heating and light bulb when needed.

Some greenhouses are equipped with a carbon dioxide sensor, as they are designed to grow plants that give higher yields at elevated levels of carbon dioxide.

Maintenance of the most optimal condition of the soil

Smart greenhouses not only provide favorable environmental conditions, but also control the condition of the soil in which the plant grows. Timely irrigation and fertilization of the plant are one of the most important conditions for maximum yield. The main task supported by all greenhouses is to measure soil moisture, which indicates whether the plant is under water voltage. In addition, it is possible to measure salinity, which gives information about fertilizers. Based on these data, irrigation systems are automatically turned on to meet the needs of plants in real time.

\section{Infection control}

This function is not common; it is most often present in farm greenhouses. Crop infection is a persistent test in agriculture, as each outbreak causes large losses in crop fields. Agrochemical treatments are available at hand, but 
farmers often do not know the best time to apply them. Using that too often causes environmental, safety and cost problems, while failure to use treatments can lead to harmful outbreaks. With the help of the machine learning platform, data on the greenhouse environment, external weather and soil characteristics reveal valuable information about the existing risks of pests and fungi. Using this information, farmers can apply treatments just when needed to ensure a healthy harvest at the lowest cost of chemicals.

Prevent theft and increase security

This function is also used exclusively in large enterprises or enterprises that grow rare and valuable crops. Greenhouses with highly valuable crops are a vulnerable target for thieves. Because traditional surveillance networks with video surveillance systems are expensive to implement, many manufacturers do not have an effective security system. In this context, IoT sensors in smart greenhouses provide an accessible infrastructure to monitor the condition of doors and detect suspicious activity. Connected to an automated alarm system, they instantly notify manufacturers when a security problem occurs.

Fig. 1 shows a block diagram of a greenhouse that has automated basic functions: watering, lighting, ventilating, cooling [2]. Sensors are connected to the microcontroller: humidity sensor, temperature, light and soil moisture. The microcontroller interrogates these sensors, collects data on the conditions of the greenhouse microclimate. If the data exceeds the limits, the microcontroller sends a signal to the relay, which closes, thus turning on the actuator under its control. When the data returns to normal, the microcontroller opens the relay and the devices turn off. Data can also be transmitted over wireless networks to cloud storage or a remote server for remote monitoring of greenhouse conditions, but we will not discuss this in this article.

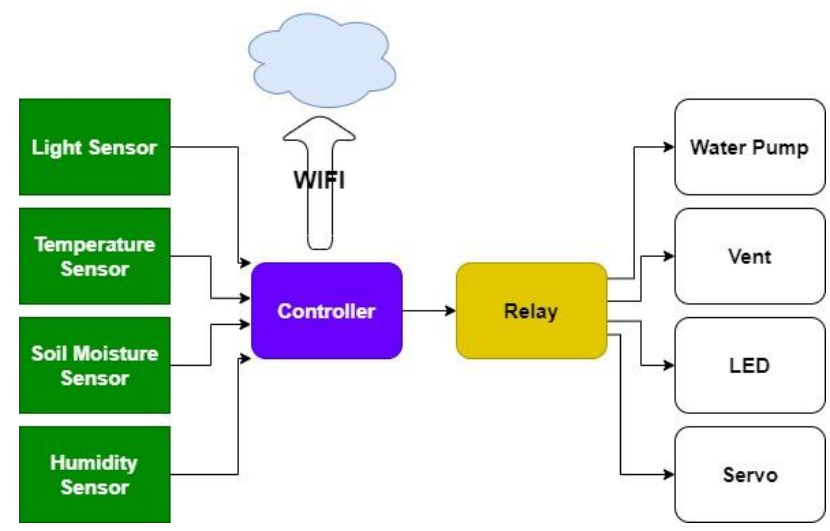

Fig. 1. Structural diagram of the smart greenhouse

The greenhouse automation algorithm is shown in Fig. 2.

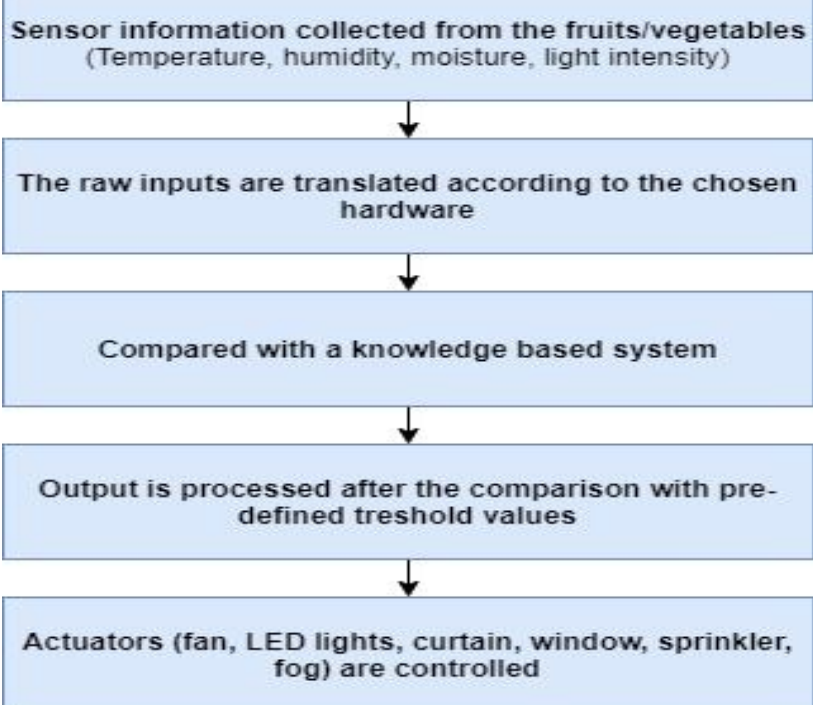

Fig. 2. Greenhouse operation algorithm

\section{SELECTING CRITERIA FOR SMART GREENHOUSE}

Greenhouse parameter monitoring and monitoring systems should collect data on greenhouse microclimate conditions 24/7. Data must be provided to the user on demand in real time. The system must automatically maintain the conditions of the microclimate and promptly respond to exceeding these limits, launching certain executive systems. An application, a bot, or a website is required to conveniently manage plant growth conditions remotely. A smart greenhouse should automate at least the basic functions: watering, lighting, ventilation and cooling.

Based on the above, we can form the following criteria for comparing and selecting these systems:

- Price

- Light control

- Soil moisture control

- Humidity control

- Temperature control

- Availability of the application

- Ventilation

\section{VI.COMPARATIVE ANALYSIS OF SMART GREENHOUSES}

There are a huge number of smart greenhouses on the market, but not all of them have the required functionality, which is described in point V. Let's compare a few of them.

- «Rozumnytsia» greenhouse automation complex is shown in Fig. 3. 


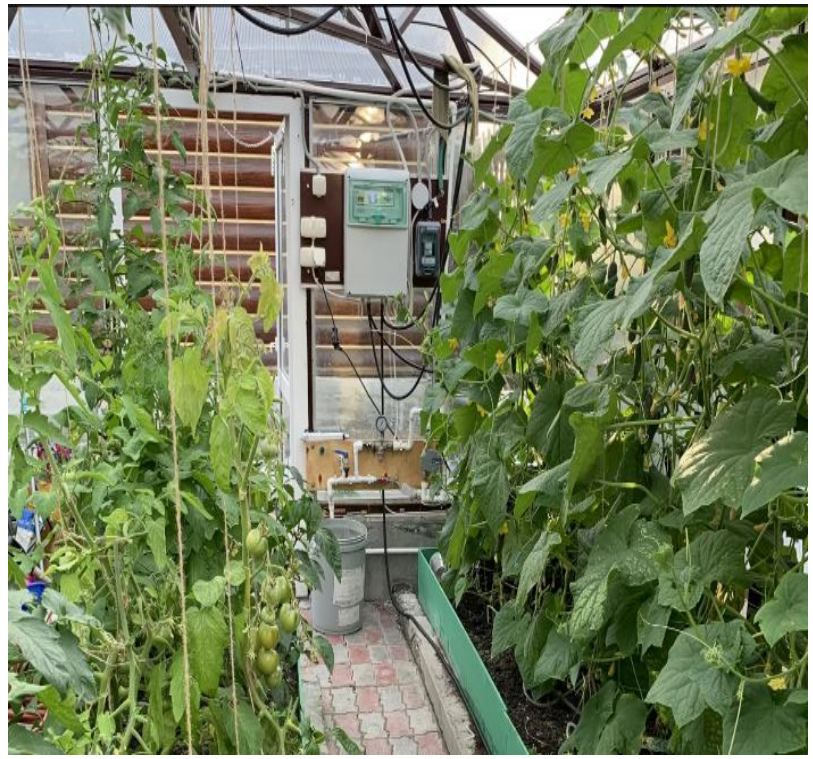

Fig. 3. «Rozumnytsia» greenhouse automation complex

This kit is designed to automate mounted greenhouses. Best suited for structures with a large area. Functionality of this greenhouse includes control of temperature and humidity of air, control of temperature and humidity of soil in 2 points of the greenhouse, control of lighting, choice of temperature of air and soil depending on time of day, inclusion of system of drip irrigation or on low indications of humidity of soil, or on a signal timer, the ability to control the humidification system, heater, light, ventilation system, control the level of the led tank [3]. The collected data is stored on the SD card, can be sent to the farmer via SMS. There are SMS notifications in case of a malfunction.

\section{Price: 12415 UAH}

Advantages: full automation of the greenhouse.

Disadvantages: this product is only a complex of automation of already mounted greenhouse. You need to make the frame yourself.

- Automatic greenhouse "Luxury" is shown in Fig. 4.

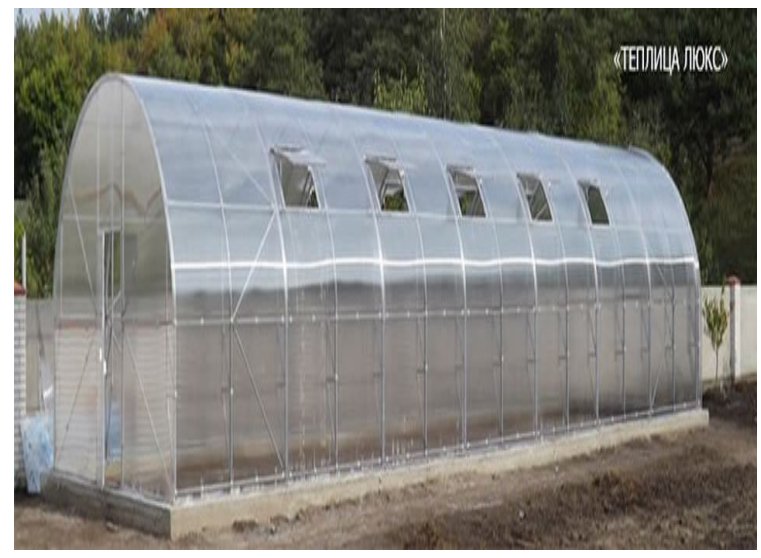

Fig. 4. Automatic greenhouse "Luxury"
This solution is perfect for growing on an industrial scale. The area of the greenhouse is $90 \mathrm{~m}^{2}$. The system is fully automated, i.e., equipped with automatic ventilation, lighting, heating and drip irrigation system. This greenhouse can be divided into 3 zones, each of which will have its own microclimate. The construction of such a greenhouse takes a month.

Advantages: automated greenhouse, low price.

Disadvantages: suitable only for farmers who grow products on a large scale, for example, for sale, lack of remote control of the greenhouse.

Price: $195139 \mathrm{UAH}$.

- Smart greenhouse "City" is shown in Fig. 5.

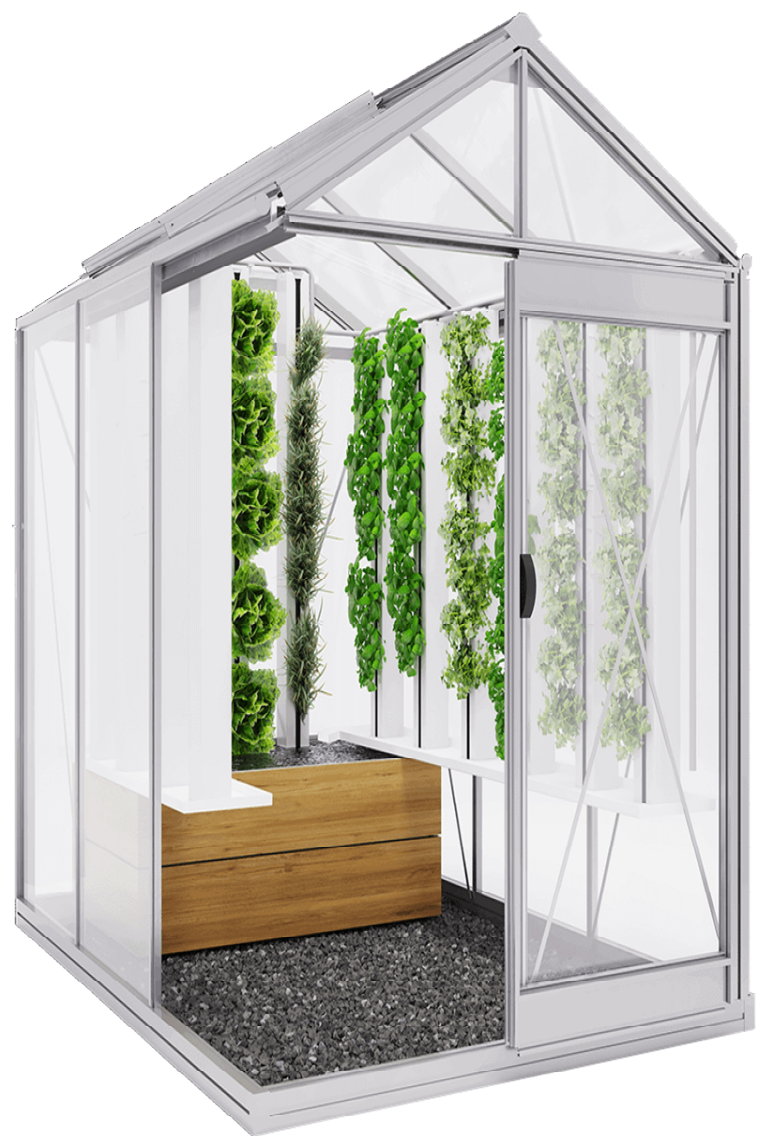

Fig. 5. Smart greenhouse "City"

This system is suitable for individual use on your own site. The frame is made of aluminum, the walls and roof are tempered glass. That is, this design is resistant to any weather. The greenhouse is fully automated, equipped with a watering and cooling system. The manufacturer provides an access to its own cross-platform application, where you one can watch all the data of the greenhouse, edit them. In addition, it is possible to communicate on the forum with other buyers, watch educational videos on the topic of farming and use the encyclopedia of the gardener.

Advantages: full automation of the system, crossplatform application with forum and media content. 

high price.

Disadvantages: no heating and lighting system, too

Price: $166,730 \mathrm{UAH}$.

- Growbox "Gin" with Gagarin phyto lamp is shown in Fig. 6.

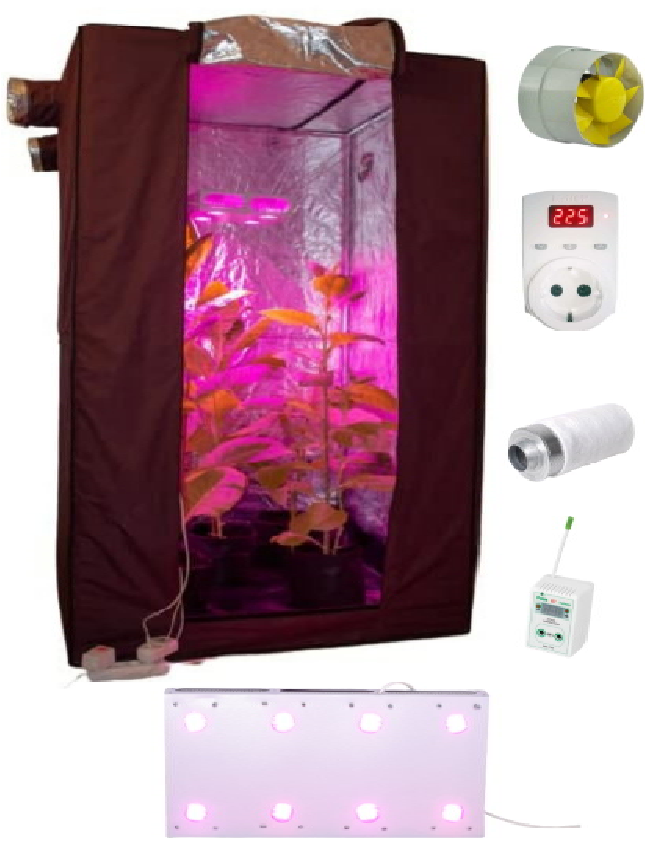

\section{Fig. 6. Growbox "Gin" with Gagarin phyto lamp}

This growbox has an aluminum frame and a ground that provides thermal insulation, but has no internal heating. Its dimensions $1.4 * 1.4 * 2 \mathrm{~m}$ allow to grow strawberries or some vegetables right in the apartment. Equipped with a system of ventilation and climate control, which allows to maintain the required temperature and humidity. To protect against spores or fungi from the environment, the ventilation system is equipped with a carbon filter [4]. The set includes a phyto lamp, which is also automatically turned on and off when needed. This solves the problem of short daylight and allows to place the greenhouse anywhere, not guided by the presence of sunlight. There is no automatic watering, which makes it impossible to leave the growbox unattended for a long time.

Advantages: small size, lighting, ventilation.

Disadvantages: high price, no watering.

Price: 15138 UAH.

- Germinator GreenCap L is shown in Fig. 7.

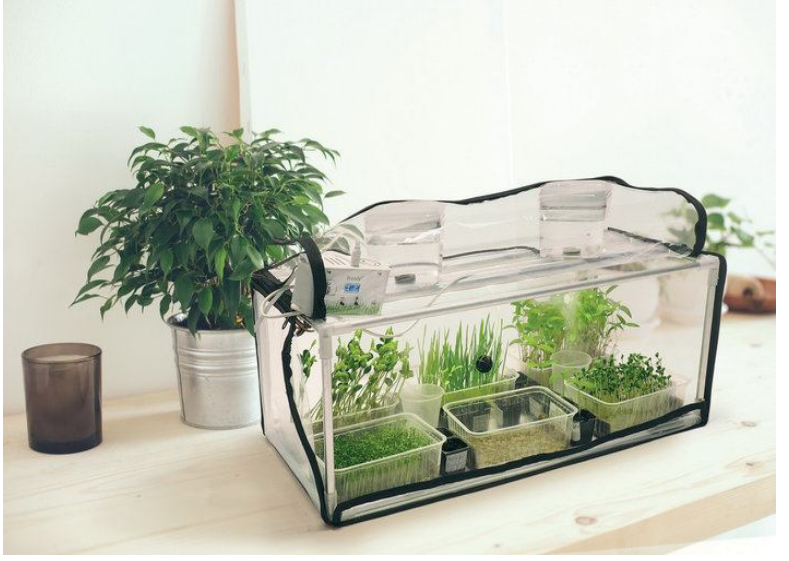

Fig. 7. Germinator GreenCap L

This type of greenhouse is the most compact of all that is on the market, easily fits even on the windowsill. Here one can grow seedlings, some vegetables or tropical plants. The germinator is able to maintain the humidity at a certain level and is equipped with two phyto lamps with LEDs of the solar spectrum, which solves the problem of a short sunny day. It has two tanks of 0.6 liters for water, which allows to humidify the air for 1-2 days. The awning is made of "flexible glass", which is able to retain heat. This design requires constant care due to the lack of watering, heating and the need to replenish the water in the tank daily.

Price: 3,919 UAH.

Advantages: small size, LEDs of the solar spectrum, humidity control.

Disadvantages: lack of heating, watering, ventilation, temperature control, frequency of water filling into the tank. Table 1 shows the comparison of different greenhouse systems comparison.

Table 1

\section{Comparison of greenhouses by main characteristics}

\begin{tabular}{|c|c|c|c|c|c|c|c|c|}
\hline & 苞 & 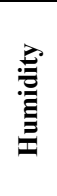 & 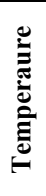 & 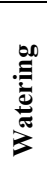 & 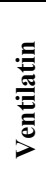 & 常 & 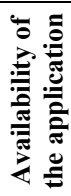 & \\
\hline "Rozumnytsia" & + & + & + & + & + & + & + & + \\
\hline "Luxury" & + & + & + & + & + & + & & + \\
\hline "City" & & + & + & + & + & & + & \\
\hline "Gin" & + & + & + & & + & & & \\
\hline GreenCap & + & + & & & & & & \\
\hline
\end{tabular}


So, comparing 5 different greenhouse systems one can clearly see that fully automated solutions are available only for large farms. In addition, only they are offered at an affordable price. Greenhouses for individual use, or mini-greenhouses for growing vegetables directly in the apartment do not have good functionality and require constant supervision of plants. Another disadvantage is the high price of such systems for meager capabilities.

\section{KEY REQUIREMENTS FOR THE DEVELOPED SYSTEM}

After analyzing the market offers, I saw that they are all divided into two types: proposals for industrial plant growing (farming) and individual use. There are no problems in the market of industrial solutions. All solutions are of high quality, have a wide range of opportunities, which allows the farmer to grow any crop and get the best results. However, there is a lack of quality systems for individual use at a reasonable price [5]. Each of the available products has a number of disadvantages. Firslty, none of them has full control over the parameters of the greenhouse microclimate. Secondly, in two of the three systems for individual use there is no control of soil moisture and watering, which is unacceptable, because timely irrigation of the plant is one of the key conditions for yield. Thirdly, smart greenhouses usually do not have remote control capabilities. In addition, all greenhouses on the market are closed solutions. They do not provide the opportunity to integrate their own ideas to increase functionality and create their own ideal system. And the last, but no less important drawback - the price. All market offers are too expensive, given all the shortcomings.

This developed product is not a ready-made smart greenhouse. This is just an automation system. Everyone can easily install it in their greenhouse. This system must have the basic functionality, namely: control of humidity, air temperature, soil moisture, light levels, automated watering, ventilation and lighting. The peculiarity of the project is the openness of the code and scalability. As a standard, my system is designed for a small growbox, which grows one flowerpot, or a mini-greenhouse, standing on the windowsill to grow several bushes of plants. However, thanks to openness, it can be easily expanded or changed to meet personal needs. The availability of remote control solves such a common problem of the need for constant supervision.

\section{STRUCTURE OF GREENHOUSE CONTROL AND MONITORING SYSTEM}

The system shown in Fig. 8 includes a microcomputer, sensors, actuators and relays to control these systems.

The microcomputer is the "brain" of the system. It surveys all sensors, collects data on the greenhouse microclimate, and, guided by established limits, turns on and off executive systems to maintain optimal plant growing conditions.

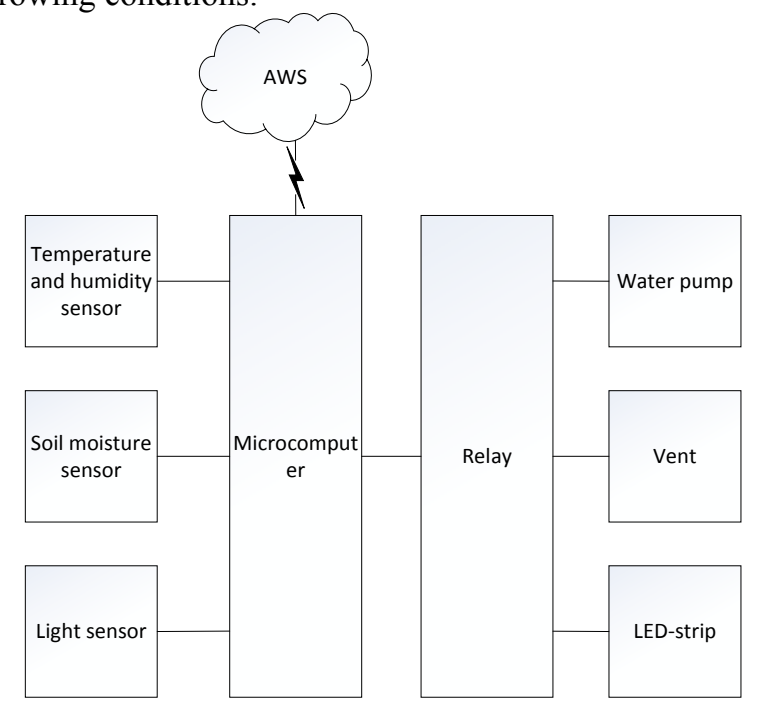

Fig. 8. Structure of greenhouse control and monitoring system

The system has sensors of soil moisture, humidity, air temperature and light level sensor to monitor the main parameters of the greenhouse.

Executive systems maintain the necessary microclimate for plants. There is a pump to automate watering, a fan to maintain the required temperature and daily ventilation (so that air does not stagnate), LED strip to provide plants with the necessary amount of light in case of insufficient natural light or short daylight.

It is impossible to control the pump or LED strip directly thanks to the microcontroller, it is impossible to connect such systems to the microcontroller. All of them are connected exclusively to the power supply and require more voltage than the microcontroller can provide. In such cases, a relay is used [6]. Relay - a switching device for closing and opening electrical circuits. Actuators are connected to the power supply via a relay, which in turn closes the circuit when it receives a signal from the microcontroller.

In addition, this system has the ability to remotely control. To do this, the user must have a configured infrastructure in AWS IoT Core and the bot in the messenger is attached to it or website. Data on plant growth conditions and the state of executive systems (on / off) are transmitted to the cloud, and from there to the user, in turn he can set thresholds for the greenhouse parameters through the user interface and enable or disable a system at its own discretion. 


\section{PRINCIPLE OF CONSTRUCTION OF HARDWARE COMPONENT OF SYSTEM}

The following hardware components have been selected for system development:

- Raspberry Pi 4 Model B microcontroller

- FC-28 soil moisture sensor

- DHT11 humidity and air temperature sensor

- BH1750 light level sensor

- Diaphragm pump

- LED strip

- Fan DE07015B12L

- Relay SRD-12VDC-SL-C

- MCP3008 ADC

The microcontroller is turned on, the synchronization node starts working, the operating system and all necessary modules are loaded. The microcontroller interrogates all sensors. Communication with the light sensor is via the $\mathrm{I} 2 \mathrm{C}$ port. $\mathrm{I}^{2} \mathrm{C}$ (IIC) - a series of asymmetric bus for communication between integrated circuits inside electronic devices. It uses two bidirectional communication lines (SDA and SCL), is used to connect low-speed peripherals to processors and microcontrollers. Data from the temperature and humidity sensor comes through a normal input port. The soil moisture sensor has two legs between which voltage passes. The wetter the soil, the lower the resistance and the higher the current. This current is fed to the comparator LM393. This comparator, guided by the voltage level, gives the value of humidity. It has outputs for analog and digital signals. Unfortunately, the Raspberry Pi microcomputer does not have analog inputs. Therefore, we can take data from the digital output of the comparator. If the soil is moist - the output will be a low signal level and vice versa. The humidity threshold is determined by a potentiometer on the comparator. However, this setting is not very accurate and does not suit us [7]. Therefore, we supply the analog output of the comparator to the ADC, which communicates with the microcontroller via the SPI port. In this way we can set the required exact humidity threshold by ourselves. After questioning these three sensors and obtaining microclimate data, the microcomputer compares this data with the set limits. If a value exceeds the limit - "raspberry" sends a control signal to the appropriate relay, which closes its circuit, including the necessary system, which will work until the parameter value returns to normal. Interestingly, this relay operates on low logic, i.e., closes the circuit if the control input signal is low. In case of insufficient soil moisture - the relay controlling the pump that will pump water for irrigation will be closed, in case of high temperature - the relay controlling the fan, in case of low light the relay through which the LED strip is connected will be closed. All three systems are shown in Fig. 9, controlled by the relay are powered by a $12 \mathrm{~V}$ voltage source.

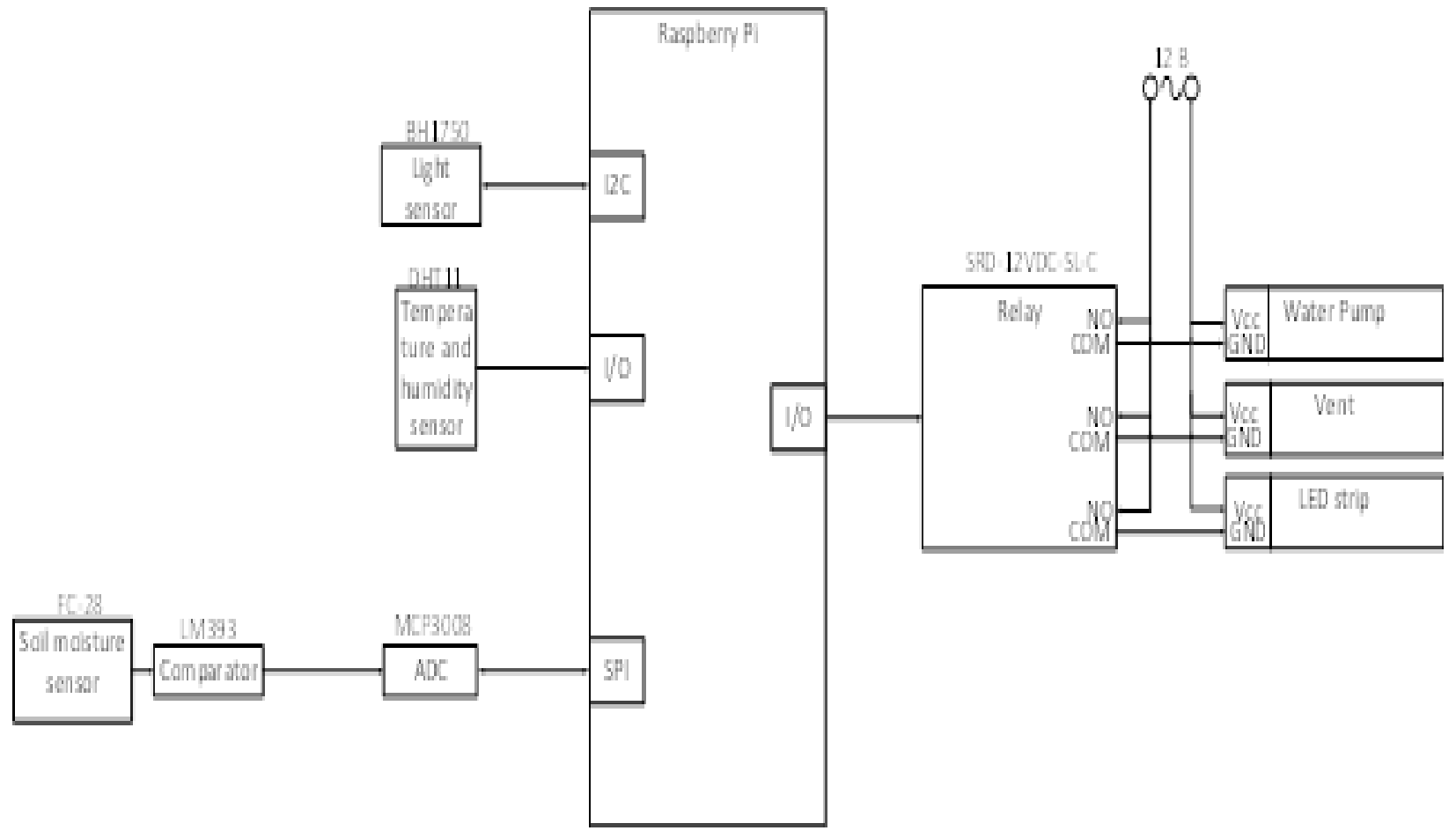

Fig. 9. Greenhouse control and monitoring system functional diagram 


\section{PROGRAM ALGORITHM OF THE GREENHOUSE MONITORING SYSTEM}

To write my own program for the system of control and monitoring of parameters in the greenhouse, I choose the Python language. This language is the best for implementing all the requirements of my system. In particular, one of the requirements is the openness and scalability of the project. Python is an intuitive enough language for anyone, regardless of programming skills, to change the system for their own special needs. Python is an interpreted, object-oriented, highlevel programming language with dynamically rigorous typing. For the last 5 years, this language has been one of the five most popular programming languages. It is able to solve a wide range of problems. The advantages of Python are flexibility, simplicity of syntax. The peculiarity of the syntax is in the selection of blocks of code by space indents. However, the language is focused on the readability of the code and its quality, as well as on ensuring the portability of programs written on it. You don't have to be a programming "guru" to write programs in a given language.

The system of control and monitoring of greenhouse parameters has the ability to remotely control using AWS cloud services. The user can view all parameters, set thresholds and enable or disable mechanisms as needed. To do this, you need to establish communication with the cloud.

The MQTT protocol is used to transfer the greenhouse data to the user and download the user-set limits to the microcomputer. MQTT is a standard messaging protocol for the Internet of Things. It is designed as an extremely lightweight messaging protocol that works on the principle of signature / publication, which is ideal for connecting remote devices with a small code size and minimal network bandwidth. Today, MQTT is used in a wide range of industries, such as automotive, manufacturing, telecommunications, oil and gas and more.

To remotely control the greenhouse, the user must have an account registered on the AWS service, a thing in the AWS IoT Core service, a table in DynamoDB, a function written in Lambda and AWS API Gateway, which is an intermediary between the lambda and the website or bot in any messenger. So, firstly, the program sets up a connection to the cloud using the MQTT protocol. Then subscribe to the publication, which contains all the values of the parameters of the greenhouse [8]. All parameters are divided into 2 types: the values of the limits, which the user sets by himself, and the values taken from the sensors, as well as available in both groups of the state of the mechanisms. User-defined settings and mechanism states are downloaded to the microcomputer. In addition, all available sensors are interviewed. Now when it has a complete picture, the system can make decisions about regulating the conditions of the microclimate. The main thing in this system is the user, so his decisions have higher priority. If the user deems it is necessary to turn on watering or ventilation - the system is sure to satisfy his desires, regardless of the existing microclimate conditions. If the user does not provide such commands, the "raspberry" compares the recorded impressions with the limit values. If any value exceeds the set limit - the appropriate mechanisms are activated. Thus, at low light levels, the LED strip will turn on, in case of insufficient soil moisture - watering, and at high temperatures, ventilation will turn on [9]. By turning these mechanisms on and off, the system changes information about their status. Captured sensor readings and new states of executive systems are formed in JSON-format, which is assigned to a variable, which in turn is sent back to the cloud, providing the user with up-to-date information about the conditions of the greenhouse microclimate [10]. Algorithm of the program of the greenhouse control and monitoring system on the basis of Raspberry Pi greenhouse automation complex is shown in Fig. 10.

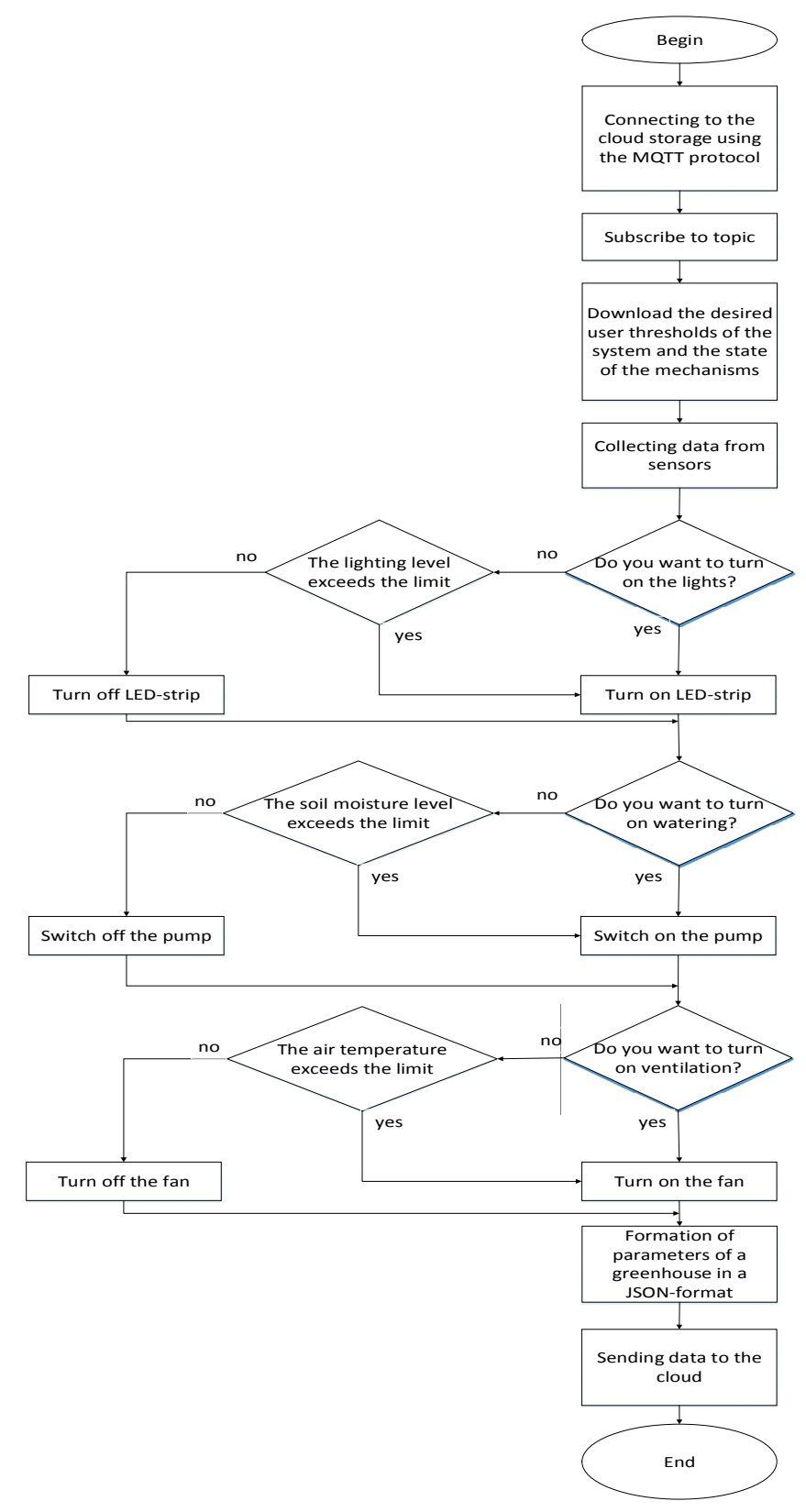

Fig. 10. Algorithm of the program of the greenhouse control and monitoring system on the basis of Raspberry Pi 


\section{XI.CONCLUSIONS}

As a result of the analysis, it was concluded that automated systems with at least basic functionality are available only to owners of greenhouse complexes or farmland. Usually, such systems are installed by specialized companies that build the necessary greenhouse from the scratch. The advantage of such systems is a reasonable price. Instead, there are no systems for individual use where the price corresponds to the quality. Such greenhouses usually have a rather small functionality. At the same time, their price is too high. Most often, they offer only control over humidity and temperature and lighting. However, even large solutions may not provide remote control, which is a significant drawback.

The advantage of the proposed solution is that it is not a ready-made greenhouse, but only a system of control and monitoring of the parameters of the greenhouse microclimate, i.e., the user can easily install it in his existing greenhouse. In addition, the solution is scalable, as it is based on the Raspberry Pi microcomputer. This board is quite powerful, so adding additional sensors or actuators will not be a problem. Another advantage of the system is that it is an Open Source.

The source code of open-source programs is available for viewing, studying and modification that allows to be convinced of absence of vulnerabilities and functions unacceptable to the user (for example, hidden tracking of the user of the program), to take part in completion of the most open program, to use the code for creation of new programs and bugs fixing, studying of the used algorithms, data structures, technologies, techniques and interfaces (as the source code can essentially supplement documentation, and in the absence of such - serves as documentation) .

\section{References}

[1] https://behrtech.com/blog/4-benefits-of-smart-greenhouses-andhow-to-get-started/

[2] https://www.researchgate.net/publication/340326857_Internet of_Things_Empowered_Smart_Greenhouse

[3] Gupta, A. K. and Johari, R. (2019). IOT based Electrical Device Surveillance and Control System, 4th International Conference on Internet of Things: Smart Innovation and Usages (IoT-SIU), pp. 1-5.

[4] Yadav, E. P., Mittal, E. A. and Yadav, H. (2018). IoT: Challenges and Issues in Indian Perspective, 3rd International Conference On Internet of Things: Smart Innovation and Usages (IoT-SIU), pp. 1-5.

[5] Sofwan, A., Sumardi, S., Ahmada, A. I., Ibrahim, I., Budiraharjo, K. and Karno, K. (2020). Smart Greetthings: Smart Greenhouse Based on Internet of Things for Environmental Engineering, International Conference on Smart Technology and Applications (ICoSTA), pp. 1-5.

[6] Lin, C. and Tseng, F. (2003). A novel micro Fabry-Perot sensor utilizing refractive index matched medium for high sensitive shear stress sensing, TRANSDUCERS '03. 12th International Conference on Solid-State Sensors, Actuators and Microsystems. Digest of Technical Pa pers (Cat. No.03TH8664), pp. 710-713.

[7] Dedeepya, P., Srinija, U. S. A., Gowtham Krishna, M., Sindhusha, G. and Gnanesh, T. (2018). Smart Greenhouse Farming based on IOT, Second International Conference on
Electronics, Communication and Aerospace Technology (ICECA), pp. 1890-1893.

[8] Singh, R. K., Berkvens, R. and. Weyn, M. (2020). Energy Efficient Wireless Communication for IoT Enabled Greenhouses, International Conference on COMmunication Systems \& NETworkS (COMSNETS), pp. 885-887.

[9] Niu, M., Zhang, D., Wang, S., Zhao, M. and. Shi, Y. (2011). Influence of the Application Rate of Organic Manure on Nitrate Migration and Accumulation in Greenhouse Soil, International Conference on Computer Distributed Control and Intelligent Environmental Monitoring, pp. 864-867.

[10] Hye, O. J., Noh, D. and Sohn, Y. (2017). Empirical test of WiFi environment stability for smart farm platform, 4th International Conference on Computer Applications and Information Processing Technology (CAIPT) pp. 1-5.

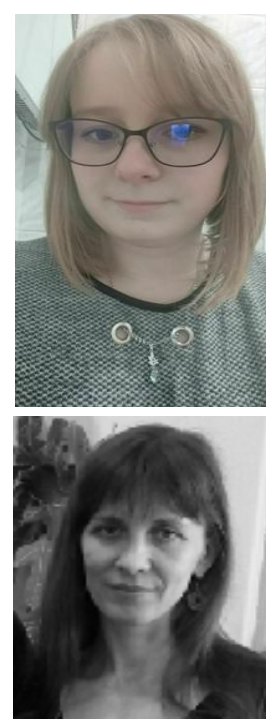

Tetiana Sahaidak an engineering student at Lviv Polytechnic National University. Her interests are artificial intelligence and IoT

Nataliia Huzynets, an engineer at Lviv Polytechnic National University. She graduated from Lviv Polytechnic National University in 2002 with a specialist degree. Areas of interest: programming, High Performance computing, Digital signal processing 